\title{
Critical Connection of Blockchain Development Platforms
}

\author{
Anshul Vyas, Leena Nadkar, Seema Shah
}

\begin{abstract}
Blockchain has gained immense popularity with success of Bitcoin. Blockchain is no longer just limited to financial sector but it has expanded its horizons far beyond it. Today innumerous Blockchain Applications are being developed and so have Blockchain Development Platforms advanced. Blockchain still being a naive technology there is excellent scope for further research in Blockchain. With these advancements, new Blockchain Development Platforms are being introduced and existing ones are rapidly modifying its features. This paper aims at comparing existing popular Blockchain Development platforms in detail with help of certain parameters to provide an aide to Blockchain Developers to choose an appropriate platform for Blockchain Development.
\end{abstract}

Keywords: Blockchain, Development Platforms, Blockchain as a service, Corda, Ethereum, Hyperledger,Multichain, Quorum

\section{INTRODUCTION}

We live in the age of technology. New technologies come up and perish but few technologies are here to stay for a long run. Back in nineties when internet gained popularity or first decade of this century when smart phones made its way in market we never thought that these technologies would become an integral part of our daily lives. Today we can say we are in same era for Blockchain, a technology all set to revolutionise industry and government. Blockchain has the power to change the way current economic and social infrastructures function and disrupt the working of many industries. It is expected that Blockchain will become a mainstream technology in next five to ten years. Hence, it is surely here for long run and a sustainable technology.Proposal of Bitcoin introduced Blockchain to the world and since then it has been gaining immense popularity. We are almost a decade old in Blockchain and innumerous platforms, development tools and services have come up for Blockchain. As per our survey very few literature pieces are available which give a detailed comparison between the existing development platforms for Blockchain. Hence, we decided to explore and compare features of existing Blockchain development platforms which will further help developers to implement Blockchain after having exact idea about the development platforms.

Revised Version Manuscript Received on July 18, 2019.

Anshul Vyas, Computer Engineering, Mukesh Patel School of Technology and Engineering, NMIMS Deemed University

Leena Nadkar, Computer Engineering, Mukesh Patel School of Technology and Engineering, NMIMS Deemed University

Dr.Seema Shah, Computer Engineering, Mukesh Patel School of Technology and Engineering, NMIMS Deemed University
The structure of the paper is as follows. The current section discusses existing issues which led to motivation behind writing this paper and contributions of the paper. Related works presents research in Blockchain and its development platforms. Blockchain overview section gives an introductory idea about Blockchain, types of Blockchain and Consensus Mechanisms. In next section, different Blockchain platforms have been discussed. Our main contribution to the paper is presented in Comparison of Blockchain Development Platforms. Plan of action discusses use of results of this paper in our current blockchain project. Conclusion and Future Work provides a summary of the proposed work.

\section{A. MOTIVATION}

As we are advancing in Blockchain, many new development platforms are coming up. Thus, making it difficult to choose appropriate development platform and type of network architecture as per the use case. According to existing records many blockchain pilot projects have been implemented for use cases which wasn't required thus causing unnecessary wastage of resources and time. Even the existing platforms today are rapidly modifying its features to stay in competition with the rest. Hence, Understanding Blockchain requirements as per the use case is essential. It is always good to compare things before starting as it gives us a clear understanding of concepts that would normally blur lines between essential and unnecessary things. Even though well established blockchain platforms have already adopted to meet needs of these new applications, more hands-on assessment on these platforms is required.

\section{B. CONTRIBUTIONS}

We have compared existing Blockchain development platforms in detail. In this paper we aim at comparing platforms Bitcoin, Ethereum, Hyperledger, Corda, Quorum, Multichain, IOTA, BigchainDB and Blockchain As Service (Azure). As per our literature survey there all the above platforms have never been compared together. We further plan to make use of this research in our current Blockchain project.

\section{RELATED WORKS}

Bitcoin paper introduced Blockchain to the world. It discussed the concepts of Longest chain been accepted in Blockchain, linking of current block to previous block using hashing algorithm. Bitcoin paper 
was also efficient in dealing with double spending problem. Incentive mechanism provides miners with incentives against Proof of work which is required to be put in by miners for process of mining. [1] Development Platforms have been introduced and discussed

[2] [3]. In [2] general overview of Ethereum, Corda , Hyperledger have been discussed and a brief has been mentioned about their consensus mechanisms too. Later comparison has been made between these platforms on basis of parameters Network type, consensus mechanism, smart contract language, access level, data storage. In [3] Blockchain case studies have been discussed and platforms like Ethereum, IBM Open Blockchain, Intel sawtooth lake, Blockstream Sidechain Elements, Eris have described on basis of parameters like Usability, Support, Documentation, Development, Limitations, Scalability, Consensus, Incentive, Currency and security and also compared on basis of it. Their result shortlists Ethereum as better platform owning to its support and documentation for developers. Whitepaper [4] discusses platforms Ethereum, Hyperledger, Corda, Quorum and IOTA as well as their features like programming languages, consensus, advantages, disadvantages.

Above papers have discussed about Blockchain platforms and compared them but none of them have compared Bitcoin, Ethereum, Hyperledger, Corda, Quorum, Multichain, IOTA, BigchainDB, Blockchain As Service (Azure) together as well as parameters for comparison have been added.

\section{BLOCKCHAIN OVERVIEW}

Blockchain is a distributed, decentralized ledger which can record transactions between multiple peers and store them permanently in an immutably and transparently. Blockchain maintains a timestamp of every Block added in the network. This technique of maintaining timestamps of all transactions was originally explained in 1971 by the group of researchers, it was intended to timestamp digital documents so that no one can backdate or tamper with them. However, it was left almost unused till Satoshi Nakamoto adapted it in 2008 to create digital currency Bitcoin. In fact, blockchain is a mix of many old technologies which have been put in a single box, this includes, Public key cryptography (1970), Cryptographic hash functions (1970), Merkle Tree (1979), Proof of work (1990), Digital Timestamps using Hash function (1991), Smart Contracts (1996), B-Money (1998).

\section{A.WORKING OF BLOCKCHAIN}

Blockchain process begins when an user requests a transaction request in its network. Transaction can be cryptocurrency, smart contract, transfer of digital assets etc. Transaction request is broadcasted to network. Blockchain network can consist of known or unknown users depending upon type of Blockchain network.

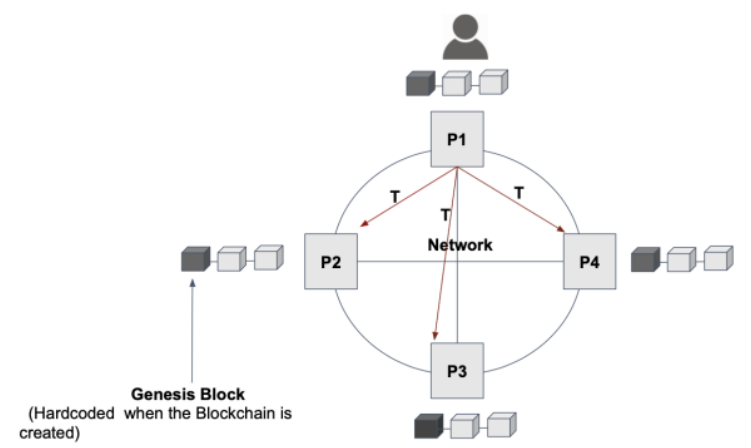

Fig. 1 Transaction request broadcasted to Blockchain network

In Fig. 1, P1 to P4 are peer nodes and $\mathrm{T}$ is the transaction which is broadcasted to the network. Received transaction is first verified and then validated by peer nodes. Once the process is successful as per the consensus followed by Blockchain network, verified transaction is combined with earlier transaction and is added to existing blockchain.

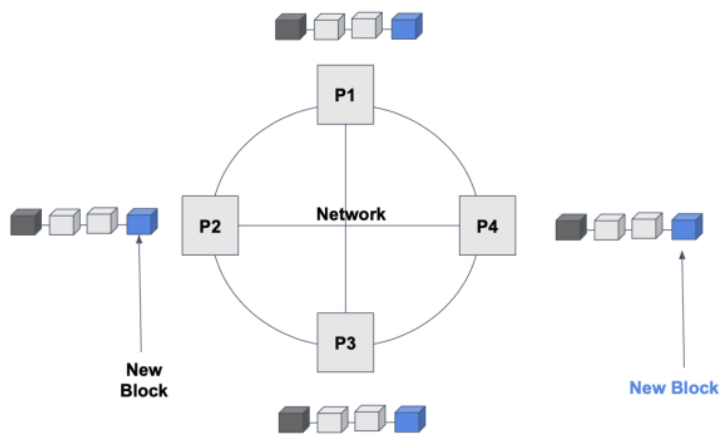

Fig. 2 New block added to existing Blockchain

In Fig.2, first block of Blockchain is called as genesis block and is hardcoded. Once new block has been added to blockchain it is said to be complete.

\section{B. TYPES OF BLOCKCHAIN}

Blockchain can be broadly divided into four categories namely Permissioned ,Permissionless, Federated and Hybrid Blockchain.

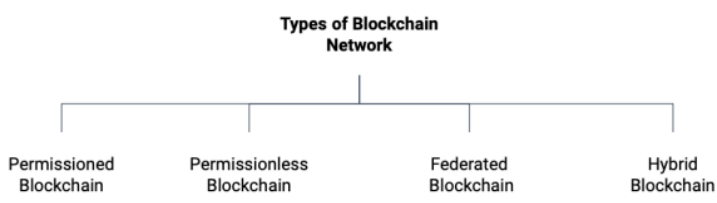

Fig. 3 Taxonomy for Types of Blockchain

Permissionless Blockchain is open and available to public. Generally, in permissionless Blockchain anonymity of users is maintained and transactions are open to everyone with view access. In Permissioned Blockchain access may be public or to limited number of peers. Here, in permissioned blockchain view \& verify access is also limited only with few peers. Federated Blockchain operates under a group or federation where access is only open to members. Hybrid Blockchain is a combination of public and private Blockchain. Blockchain is ideally suitable when multiple peers are interacting with each other. All the peers don't need to know entire interaction among rest of the peers completely. Few peers can

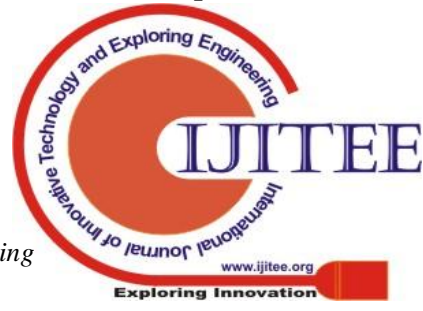


be decision makers while few are only allowed to view data. As data is on Blockchain it is going to be immutable and transparent hence, in hybrid blockchain we can maintain data on different channels and peers are given different access levels to channels as per the necessity.

\section{CONSENSUS MECHANISMS}

Consensus means a general agreement between peers in Blockchain network. As there is no centralized authority, decision can be made in decentralized environment using consensus mechanisms. For Blockchain, some of popular consensus mechanisms are Proof of Work, Proof of Stake,

Proof of Burn, Proof of Elapsed Time. In Proof of Work (POW) some work needs to be done to acquire a new block. This is done by miners. Proof of work is not energy efficient as solving hash codes requires tremendous amount of electrical energy. Hence, Proof of Stake (POS) was proposed. In POS, peer needs to acquire sufficient stake in Blockchain network to validate a blockchain. For, Proof of Burn(POB) peer needs to burn a predefined part of his wealth to mine a new block. In Proof of Elapsed time (POE) each participant in Blockchain network waits for a random amount of time and the first peer to finish becomes leader for new block.

\section{SMART CONTRACTS AND ORACLES}

Smart contract runs on top of Blockchain, when two or more interacting peers agree on a smart contract computer code. It is a set of rules to which interacting peers need to agree. Digital assets are accessed, distributed and used by the peers as per the pre-defined contract among them. All the information regarding legal term in contract needs to be given while coding.

An oracle is an agent that finds and verifies real-world occurrences and submits this information to a blockchain so that it can be used by smart contracts.

\section{OVERVIEW OF BLOCKCHAIN DEVELOPMENT PLATFORMS}

After the success of Bitcoin, Blockchain popularity grew immensely. With advancement in Blockchain many Blockchain Development Platforms emerged. Some of popular Blockchain Development platforms are Bitcoin, Ethereum, Hyperledger, Corda, Quorum, Multichain, IOTA, BigchainDB, Blockchain As Service (Azure) and many more. In following description of Blockchain platforms they have been discussed on basis of their overview, consensus mechanism, type of network architecture, smart contracts and development.

\section{A. Bitcoin}

Bitcoin paper proposed a decentralized currency which can be sent between two peers without interference of a third party or centralized authority. Bitcoin solved the problem of double spending in a decentralized environment efficiently.
Bitcoin uses Proof of work consensus mechanism. Proof of work means that miners need to spend huge amount of electricity and perform complex calculations to validate and add a block to Blockchain. Bitcoin has been secure as the chain has become long with time and attacking or changing any information in Bitcoin chain will require extremely huge amount of recalculation of hash which may surpass the profit gained by the hacker. Hence, currently Bitcoin is secure. Bitcoin is public blockchain, anyone can join the network. Transactions in bitcoin are transparent but identity of peers is kept anonymous. Bitcoin protocol can be used to create smart contracts. Bitcoin has been written in $\mathrm{C}++$ language. Bitcoin scripting language is used for embedded input and output for bitcoin transactions.

\section{B. Ethereum}

Ethereum platform enabled people to look at Blockchain beyond Bitcoin. It gave an user friendly platform to code decentralized applications. There is no interference of third party in these decentralized applications. Currently Proof of Work is used as consensus mechanism for Ethereum but they will gradually move to Proof of stake which means one can participate in process of mining at cost of a certain stake. This can work as efficient method instead of Proof of work as it will help reduce tremendous amount of electricity being used for Proof of work and even ensure transparency in chain as only a peer with certain amount of stake can participate in mining. Hence, if peer tries to hack or change records on Blockchain will face a significant amount of loss too. Thus, they will be discouraged to do any such attack on Ethereum Blockchain thus assuring its safety. Ethereum Blockchain is public in nature. Smart contracts are executed on decentralized system called Ethereum Virtual Machine. Applications of Ethereum run without a possibility of downtime, censorship, fraud or third party interference. Currently Ethereum is most popular platform for writing decentralized applications and developers of Ethereum have even claimed to be future of Internet. Ethereum has generalized protocols and easy language and has become a platform thriving with use cases for decentralized applications. Coding for Ethereum smart contracts is done using Solidity, Serpent and LLL languages. Ethereum also used frameworks for Development, popular framework being Truffle. Ethereum is Turing Complete. Also, Ethereum Community has set goals for Ethereum development deliverables of which have been delivered on time till date. It has fault tolerance and maintains stable state even if few machines in network are down.

\section{Hyperledger}

Hyperledger ecosystem is based on solution providers and users focussed on Blockchain related use cases that will work across a variety of industrial sectors. It has a collection of open source projects. Currently it is being led by Linux Foundation. Hyperledger project has various frameworks under its license like Fabric, Sawtooth, Burrow, Iroha and Indy. Hyperledger also

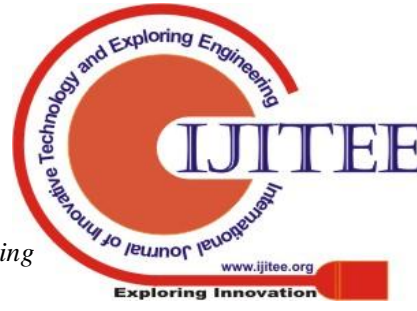


maintains modules, a suite of software used for deploying and maintaining Blockchains, examining data on ledger and designing prototyping and extending Blockchain networks. Hyperledger can work as private or permissioned Blockchain. Hyperledger Fabric is meant to be a foundation for developing Blockchain Applications with a modular architecture. It can be programmed using Chaincode or Java and it follows Kafka Consensus mechanism which is permissioned voting based. Hyperledger Sawtooth utilizes modular platform for building, deploying and running distributed ledgers. It follows Proof of Elapsed model consensus mechanism which allows it to promise scalability of Bitcoin Blockchain without high resource consumption. Hyperledger Burrow is a permissioned Ethereum smart contract blockchain system. It follows Proof of Stake Tendermint consensus engine and coding languages are Solidity, Serpent. LLL. Hyperledger Iroha is designed for mobile application development with libraries for Android

and iOS. It follows Proof of Stake Tendermint consensus engine and coding language used is solidity, Serpent, LLL. Hyperledger Indy aims to give individuals ownership of their identities. It provides tools, libraries and reusable components for providing digital identities on blockchain. It follows RBFT consensus mechanism and coding languages used are .Net, Java, Python, Objective C.

\section{Corda}

Corda has been created by R3, it is a consortium financial institutions. Initially, Corda was proposed for financial services. It automates legal agreements between businesses and solves issues of duplicated inconsistent data and faulty business logic. There are two types of Consensus mechanisms in corda, Validity Consensus and Uniqueness consensus. Validity consensus is checked by each signeer before they sign transaction whereas Uniqueness consensus is only checked by notary service. However, Corda is not being considered as Blockchain solution but as a Distributed Ledger Technology. Corda is open source enterprise blockchain. Development of Corda can be done in Kotlin or Java language. Innumerous and diverse applications can interact on a global level on a single platform of Corda.

\section{E. Quorum}

Quorum has been created by JPMorgan. We can see to it as permissioned Ethereum. It is open sourced and permissioned implementation of Blockchain. It has transaction level privacy i.e it uses shielded transactions. It follows Quorum Chain consensus, which is a time based majority voting algorithm. It consists of a smart contract to govern participation of peers in consensus, ethereum transactions to propagate vote through network and ethereum signature validation to validate maker and voter nodes. Block creation is only allowed by maker node. Development for Quorumchain is done using solidity and constellation.

\section{F. Multichain}

Multichain is being used to build applications on Blockchain and maintaining shared ledgers for applications. It is a private Blockchain. Multichain provides API and Command line interface. Mining is done in Multichain using Proof of Work as consensus mechanism. A Multichain is established when two Blockchain nodes connect with each other. In version 2.0 of Multichain Smart filters have been introduced. Smart filters allows defining of customized rules to check validity of transactions. Multichain supports two types of smart filters namely transaction and stream filters. In transaction smart filters input, output and metadata is examined and in stream filters on and off chain data is validated together. Smart filters can be developed using Javascript. Multichain has been written in $\mathrm{C}++$ but it provides JSON-RPC which can be used with modern programming languages.

\section{G. IOTA}

IOTA can be described as a decentralized platform for transactions between devices connected to internet. It is a distributed ledger technology which can be used for Blockchain but similar to corda, IOTA too isn't considered as Blockchain. IOTA network is called Tangle. Instead of forming blocks Tangle forms distributed ledger like web of information. IOTA is an open source project. IOTA differs from Blockchain in two things Directed Acyclic Graph, it forms tangle, primary element on which IOTA depends and second is working of consensus mechanism. In IOTA every peer in network is part of decision making process of consensus mechanism. IOTA development is possible in languages like C, Go, Java, Javascript.

\section{H. BigChainDB}

BigChainDB is an open source blockchain scalable database. BigChainDB uses Mongo Database in backend for database storage. They store blockchains inside the distributed database. It has immutable data storage. Once data has been written to BigChainDB it cannot be modified. It has query API Engine to query BigChainDB. BigChainDB uses Byzantine Fault Tolerance as Consensus Mechanism. It has customizable Network type allowing it to be used as Public or Private network as per the need of Application. BigChainDB supports both traditional and blockchain stack. It doesn't have any native currency.

\section{Blockchain as a service}

In Blockchain as a service cloud based solutions are used to build, deploy and use blockchain applications and smart contracts. At times, Blockchain as a Service leads to increased technical complexities and operational overhead. Blockchain as Service handles backend for customer or business. Currently, Blockchain as a service is being offered by platforms like Microsoft Azure, Amazon, Oracle, IBM Blockchain. Infrastructure and Performance issues of Blockchain are taken care by Blockchain as a service provider. 


\section{COMPARISON OF BLOCKCHAIN DEVELOPMENT PLATFORMS \& RESULTS}

In this paper we will be comparing Blockchain Development Platforms on the basis of Type of network architecture, Consensus Mechanism, Smart Contracts, Currency, Governance, Data storage, Development Languages, Support and Documentation, Github repository, Incentive mechanism, security, framework, ports , turing complete development platform.

Bitcoin and Ethereum are public in nature. Hyperledger is permissioned network. Corda, IOTA and Quorum are permissioned private. Multichain is private. BigChainDB and Blockchain as a service (Baas) can be public or private.

Bitcoin and Multichain use Proof of work as consensus mechanism. Ethereum currently uses Proof of Work but will soon adapt Proof of stake as its consensus mechanism. Hyperledger and BigChainDB have Byzantine fault tolerance as its consensus mechanism. Corda uses Validity and Uniqueness consensus. Quorum uses its own consensus mechanism Quorumchain. In IOTA every peer in network participates in consensus mechanism.

Bitcoin, Ethereum, Hyperledger, Corda, Quorum, Baas support Smart Contracts. Multichain has Smart filters instead of smart contracts. IOTA doesn't support smart contracts.

Bitcoin has its own currency, success of which led to popularity of Blockchain. Currency of Ethereum is Ether. Other platforms don't have their currency.

Bitcoin and Ethereum are open source public blockchain. Hyperledger is governed by Linux Foundation, Corda by R3 Consortium. Quorum is backed by JP Morgan. Blockchain as a service has been started by various organizations like Amazon, Microsoft, IBM, Oracle.

Swarm is used by Ethereum for Data Storage and Hyperledger uses Couch and Level DB.

Bitcoin uses Bitcoin Script for development. Solidity, LLL, Serpent is used by Ethereum, Hyperledger Burrow and Hyperledger Iroha. Hyperledger Fabric uses Go Chaincode, Java while Hyperledger Sawtooth has Python and Hyperledger Indy can use .Net, Java, Python, Objective C, Rust. Corda uses modern development languages like Kotlin, Java. Quorum uses Solidity and constellation. While Multichain and IOTA use Javascript.

Incentive Mechanism is only there for Bitcoin and Ethereum not for other Blockchains considered for comparison in this paper.

Truffle and Dribble are popular frameworks for Ethereum while Hyperledger Fabric, Sawtooth, Burrow, Iroha and Indy are the ones for Hyperledger. All the Blockchain Platforms compared in this paper are active currently with support, documentation and github repository available for all of them.

Ethereum works on TCP port 8545 while Hyperledger works on port 7053. Bitcoin is not Turing Complete whereas other Development Platforms Ethereum, Hyperledger, Corda and Quorum are turing complete.

Table I : Major Comparison Parameters among Blockchain Development Platforms

\begin{tabular}{|c|c|c|c|c|c|}
\hline \multirow{2}{*}{\begin{tabular}{|c|} 
Blockchain \\
Development \\
Platforms
\end{tabular}} & \multicolumn{5}{|c|}{ Parametres for comparison } \\
\hline & $\begin{array}{c}\text { Type of Network } \\
\text { Architecture }\end{array}$ & Governance & Consensus & Smart Contracts & $\begin{array}{c}\text { Development } \\
\text { Languges }\end{array}$ \\
\hline Bitcoin & Public & & Proof of Work & Yes & Bitcoin Script \\
\hline Ethereum & Public & $\begin{array}{l}\text { Ethereum } \\
\text { Developers }\end{array}$ & \begin{tabular}{|l|} 
Proof of Work, \\
Proof of Stake
\end{tabular} & Yes & $\begin{array}{c}\text { Solidity, LLL, } \\
\text { Serpent }\end{array}$ \\
\hline Hyperledger & $\begin{array}{l}\text { Permissioned, } \\
\text { Private }\end{array}$ & $\begin{array}{l}\text { Linux } \\
\text { Foundation }\end{array}$ & $\begin{array}{c}\text { Byzantine Fault } \\
\text { Tolerance }\end{array}$ & Yes & $\begin{array}{c}\text { Go, Chaincode } \\
\text { Java (Fabric) } \\
\text { Python } \\
\text { (Sawtooth) } \\
\text {.Net, Java, } \\
\text { Python, } \\
\text { Objective C, } \\
\text { Rust (Indy) } \\
\text { Solidity, } \\
\text { Serpent, LLL } \\
\text { (Burrow) } \\
\text { Solidity, } \\
\text { Serpent, LLL } \\
\text { (Iroha) }\end{array}$ \\
\hline Corda & $\begin{array}{c}\text { Permissioned, } \\
\text { Private }\end{array}$ & R3 & $\begin{array}{c}\text { Validity } \\
\text { Uniqueness } \\
\text { Consensus } \\
\end{array}$ & Yes & Kottin, Java \\
\hline Quorum & \begin{tabular}{|c|} 
Permissioned, \\
Private
\end{tabular} & JP Morgan & \begin{tabular}{|c|} 
Quorum Chain \\
Consensus
\end{tabular} & Yes & $\begin{array}{c}\text { Solidity, } \\
\text { Constellation }\end{array}$ \\
\hline Multichain & Private & Multichain & Proof of Work & Smart Filters & Javascript \\
\hline IOTA & $\begin{array}{c}\text { Permissioned, } \\
\text { Private }\end{array}$ & IOTA & $\begin{array}{l}\text { Every node } \\
\text { participates }\end{array}$ & $\begin{array}{c}\text { Doesn't support Smart } \\
\text { contracts }\end{array}$ & $\begin{array}{l}\text { Java, } \\
\text { JavaScript }\end{array}$ \\
\hline BigChain DB & $\begin{array}{c}\text { Public or } \\
\text { Private }\end{array}$ & BigChainDB & $\begin{array}{c}\text { Byzantine Fault } \\
\text { Tolerance }\end{array}$ & & \\
\hline $\begin{array}{c}\text { Blockchain As A } \\
\text { Service }\end{array}$ & $\begin{array}{l}\text { Public or } \\
\text { Private }\end{array}$ & $\begin{array}{c}\text { Amazon, } \\
\text { Microsoft, IBM, } \\
\text { Oracle }\end{array}$ & & Yes & \\
\hline
\end{tabular}

\section{PLAN OF ACTION}

Currently, we are working on Blockchain project and working on Use cases for KYC Updation, Education Blockchain and Peer to peer Charity Blockchain. After having surveyed the platforms above and comparing them as per the above mentioned parameters, we plan to make use of the research for our project. KYC Updation requires involvement of multiple financial institutions and customer where all don't need to know entire information about each other. Also, Blockchain is limited to few peers. It doesn't need to be public in nature, one should be allowed to join blockchain only if authorized. Hence, private blockchain like Hyperledger can prove effective at such stage. Whereas in case of Education and Peer to peer charity, data needs to be accessible to public and anyone can join the network. Hence, for these use cases we plan to use a blockchain which is public in nature like ethereum.

\section{CONCLUSION}

Blockchain is a technology that holds power to change working of current of industries. Records can only be appended in Blockchain as it is immutable. Modification or deletion of records is not permitted. Thus, Blockchain is transparent and secure. With advancement of Blockchain, new Blockchain development platforms and features came up. At such time comparison between development platforms gives a better clarity while choosing Blockchain Development platform.

After surveying different Blockchain platforms we can conclude that, selection of development platform depends upon the type of Use case. In this paper we have given a detailed comparison between popular Blockchain

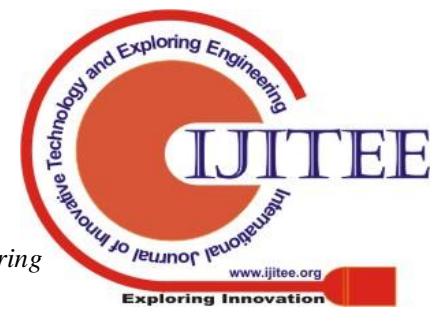


development platforms existing today. As per the use case, developer can compare the platforms with help of comparison table and choose platform according to language preference or type of suitable network architecture.

We further intend to study use cases domains and map development platforms with network architecture and use case domains. Also, study and research business problems where Blockchain plays and important role and where it can act as an overhead, thus helping developers and others to have a picture in mind about when and which development platform to use for a particular business problem.

\section{REFERENCES}

1. Satoshi Nakamoto, "Bitcoin: A Peer-to-Peer Electronic Cash System",2008

2. Chinmay Saraf and Siddharth Sabadra, "Blockchain Platforms : A Compendium", International Conference on Innovative Research and Development, IEEE, May 2018

3. M.Macdonald, L. Liu- Thorrold and R.Julien, "The Blockchain : A Comparison of Platforms and Their Uses Beyond Bitcoin”, 2017

4. Ben Wald and Bill Brock, "The innovator's guide to picking the right Blockchain”, VERY, 2017

5. Tong Wu,Xiubo Liang, "Exploration and Practice of Inter Banking Application based on Blockchain",IEEE 12th International conference on Computer Science \& Education, August 2017

6. Maxim Amelchenko, Shlomi Dole, and Ben Gurion, "Blockchain Abbreviation implemented by message passing \& shared memory", IEEE, 2017

7. Masashi Sato and Shin'ichiro Matsuo, "Long-term public blockchain: Resilience against compromise of Underlying Cryptography",2nd IEEE European Symposium on Security and Privacy Workshops, EuroS and PW., 2017

8. Suporn Pongnumukul et.al., "Performance Analysis of Private Blockchain Platforms in Varying Workloads", 2017 26th International Conference on Computer Communications and Networks, ICCCN., 2017

9. Ingo Weber et.al., "On Availability of Blockchain-Based Systems", Proceedings of the IEEE Symposium on Reliable Distributed Systems.,2017

10. Harish Sukhwani et.al., "Performance Modelling of PBFT Consensus Process for Permissioned Blockchain Network (Hyperledger Fabric)",Proceedings of the IEEE Symposium on Reliable Distributed Systems.,2017

11. Ashiq Anjum, Manu Sporny and Alan Sill, "Blockchain Standards for Compliance and Trust”,IEEE Cloud Computing., August 2017,vol. 4, pp. 84-90.

12. Ning Zhou, Menghan Wu and Jianxin Zhou, "Volunteer Service Time Record System Based on Blockchain Technology ",Proceedings of 2017 IEEE 2nd Advanced Information Technology, Electronic and Automation Control Conference, IAEAC, 2017

13. Henry Kim and Marek Laskowski, "A Perspective on Blockchain Smart Contracts", Computer Communication and Networks (ICCCN), 2017 26th International Conference.,September 2017

14. Haneffa Muchlis Gazali et.al., "Re-inventing PTPTN Study Loan With Blockchain and Smart Contracts",Information Technology (ICIT), 2017 8th International Conference, October 2017
15. Heng Hou, "The Application of Blockchain Technology in E-government in China",Computer Communication and Networks (ICCCN), 2017 26th International Conference, September 2017

16. Darra L.Hofman, "Legally Speaking: Smart Contracts, Archival Bonds, and Linked Data in the Blockchain", Computer Communication and Networks (ICCCN), 2017 26th International Conference, August 2017

17. Imran Bashir, Mastering Blockchain, Packt, March 2017

18. Narayan Prusty, Building Blockchain Projects, Packt, April 2017

19. Deepak K.Tosh et.al., "Consensus Protocols for Blockchain based Data Provenance: Challenges \& Opportunities", Ubiquitous Computing, Electronics and Mobile Communication Conference (UEMCON), 2017 IEEE 8th Annual., January 2018,pp. 469-474.

20. Muhamed Turkanovic et.al, "EduCTX : A blockchain based higher education credit platform", IEEE, 2017 